\title{
Outcomes of extended duration therapy for drug-susceptible cavitary pulmonary tuberculosis
}

\author{
Hyun Lee ${ }^{1}$, Jang Won Sohn ${ }^{1}$, Yun Su Sim ${ }^{2,3}$, Tae Rim Shin ${ }^{2,3}$, Dong-Gyu Kim ${ }^{2,3}$, Hayoung Choi ${ }^{2,3}$ \\ ${ }^{1}$ Division of Pulmonary Medicine and Allergy, Department of Internal Medicine, Hanyang University College of Medicine, Seoul, Korea; ${ }^{2}$ Division \\ of Pulmonary, Allergy, and Critical Care Medicine, Department of Internal Medicine, Hallym University Kangnam Sacred Heart Hospital, Seoul, \\ Korea; ${ }^{3}$ Lung Research Institute, Hallym University College of Medicine, Chuncheon, Korea \\ Contributions: (I) Conception and design: H Lee, H Choi; (II) Administrative support: H Choi; (III) Provision of study materials or patients: TR Shin, \\ DG Kim; (IV) Collection and assembly of data: H Lee, JW Sohn; (V) Data analysis and interpretation: H Lee, YS Sim, H Choi; (VI) Manuscript \\ writing: All authors; (VII) Final approval of manuscript: All authors. \\ Correspondence to: Hayoung Choi. Division of Pulmonary, Allergy, and Critical Care Medicine, Department of Internal Medicine, Hallym University \\ Kangnam Sacred Heart Hospital, Seoul, Korea. Email: hayoung.choi@hallym.ac.kr.
}

\begin{abstract}
Background: Extending the continuation phase treatment duration is recommended to improve outcomes of drug-susceptible cavitary pulmonary tuberculosis (TB), but limited data are available on extended treatment outcomes.

Methods: We evaluated outcomes of 67 patients with drug-susceptible cavitary pulmonary TB who had received extended therapy. The primary endpoint of our study was the rate of a favorable outcome (cured or treatment completion without recurrence).

Results: Of the 67 patients, 40 (59.7\%) were culture negative and 27 (40.3\%) were culture positive two months after treatment initiation. The median treatment duration was 275 days. Extended duration therapy resulted in a $100 \%$ treatment success rate and $2.5 \%$ recurrence rate in patients with a negative culture at month 2 . However, patients with a positive culture at month 2 , showed a $74.1 \%$ treatment success rate and $8.0 \%$ recurrence rate $(\mathrm{P}<0.001$ and $\mathrm{P}=0.554$, respectively). In multivariable analyses, positive culture at month 2 was associated with greater odds of unfavorable outcomes (adjusted OR, 17.04, 95\% CI, 1.68-177.92).

Conclusions: While extending the continuation phase was associated with favorable outcomes in pulmonary TB patients with negative culture at month 2, the same could not be achieved in those with positive culture at month 2 , suggesting that this condition might not be overcome by merely extending the continuation phase.
\end{abstract}

Keywords: Pulmonary tuberculosis (pulmonary TB); cavitation; two-month culture; treatment outcome

Submitted Nov 28, 2019. Accepted for publication Feb 05, 2020.

doi: $10.21037 /$ atm.2020.02.104

View this article at: http://dx.doi.org/10.21037/atm.2020.02.104

\section{Introduction}

Most patients with drug-susceptible tuberculosis (TB) are successfully treated using the standard six-month treatment regimen consisting of isoniazid, rifampin, ethambutol, and pyrazinamide, with a treatment success rate of 90-95\% (1). Previous studies have shown that treatment outcomes can be suboptimal in patients with additional risk factors, such as the following: being $>10 \%$ below ideal body weight; being an active smoker; and having diabetes mellitus, human immunodeficiency virus (HIV) infection, or signs of extensive disease observed on chest radiographs (2-7).

Besides the above factors, the most well-known risk factors for unfavorable treatment outcomes are cavitation on chest radiograph (3) and positive culture 2 months posttreatment initiation (hereafter positive culture at month 2) $(3,8)$. In patients treated for six months, having both cavitation and positive culture after a two-month treatment 


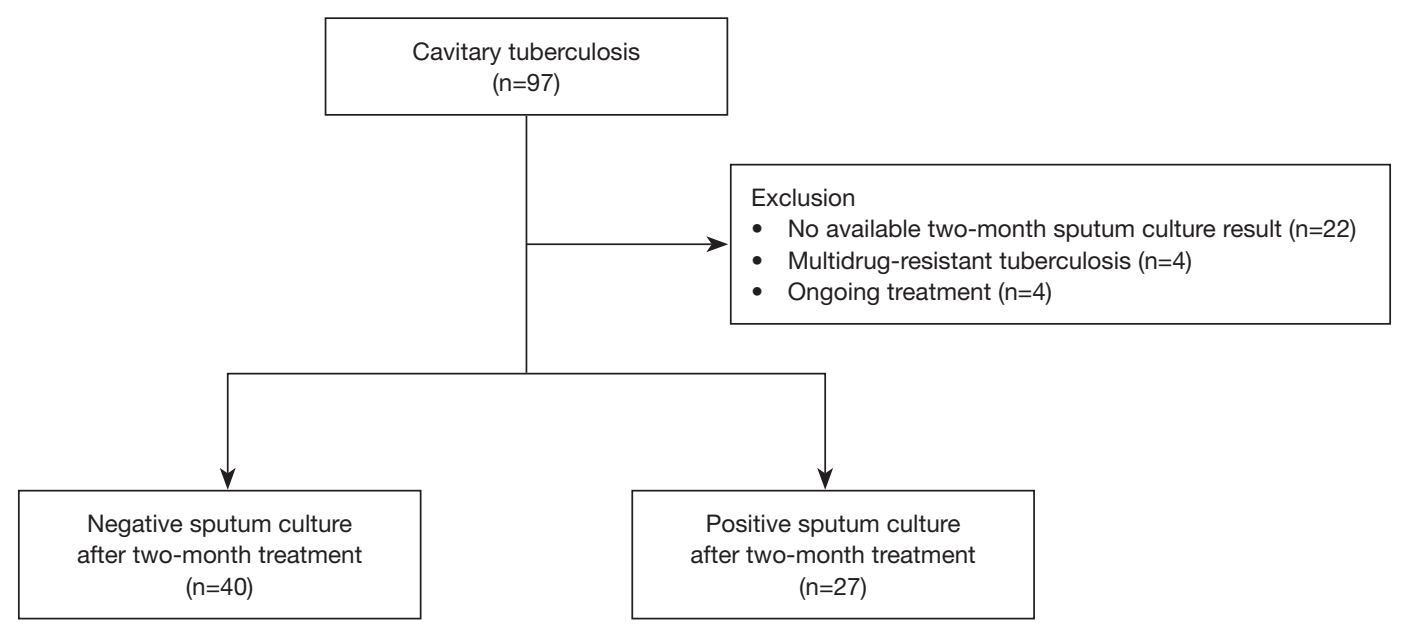

Figure 1 Flow chart of the study population.

is associated with an approximately $20 \%$ recurrence rate (7-9). Accordingly, the current guidelines have proposed extending the continuation phase of treatment with isoniazid and rifampin for an additional three months in these patients, corresponding to a total of nine months of therapy. It is also recommended to consider extending the continuation phase in patients with cavitation only (7). However, there is limited treatment outcome data available to support this recommendation.

Accordingly, we aimed to investigate the treatment outcomes in patients with drug-susceptible cavitary pulmonary TB who received treatment with an extended continuation phase.

\section{Methods}

\section{Study population}

We included all consecutive adult patients (age $\geq 18$ years) with newly diagnosed or retreated drug-susceptible cavitary TB at Hallym University Kangnam Sacred Heart Hospital (a 577-bed referral hospital in Seoul, South Korea) between January 2012 and June 2017. During the study period, 1,288 patients were diagnosed with pulmonary $\mathrm{TB}$, while $97(7.5 \%)$ were diagnosed with cavitary pulmonary TB. After excluding patients with no available two-month sputum culture results $(n=22)$, those initially diagnosed with multidrug-resistant TB (MDR-TB) $(n=4)$, and those with ongoing treatment $(n=4), 67$ patients were included (Figure 1). Before commencing TB treatment, the attending physicians determined the baseline comorbidities. The study protocol was approved by the Institutional Review Board (IRB) of Hallym University Kangnam Sacred Heart Hospital (IRB application no. 2018-07-007). All data were anonymized before analysis and the need for written informed consent was waived by the IRB due to the retrospective nature of this study.

\section{Microbiologic and radiologic examination}

Acid-fast bacilli (AFB) staining was performed on sputum samples with an auramine-rhodamine fluorescent stain, followed by confirmation with Ziehl-Neelsen-staining. Staining results were graded according to the American Thoracic Society/Centers for Disease Control and Prevention guidelines (10). Specimens in which the AFB smear results were categorized as grades $\geq 1+$ were defined as smear positive. All clinical specimens were cultured on both solid and liquid media for six weeks. To this end, decontaminated samples were inoculated into mycobacterial growth indicator tubes (MGIT 960 system; Becton Dickinson, Sparks, MD) and 3\% Ogawa agar (Shinyang, Seoul, Korea). All positive cultures were subjected to an $\mathrm{AFB}$ smear to confirm the presence of AFB and exclude contamination. Pulmonary TB was diagnosed based on sputum AFB smear or Mycobacterium tuberculosis (MTB) polymerase chain reaction (PCR) results. The PowerChek MTB/NTM Real-time PCR Kit (Kogene Biotech, Korea) was used to analyze sputum samples. Drug susceptibility testing (DST) was performed using the absolute concentration method with the Lowenstein-Jensen medium at the Korean Institute of Tuberculosis (11). Follow-up 
sputum AFB smear and culture were performed monthly. All the procedures were performed in Class 2 biosafety cabinets.

With regard to the chest radiograph, the posteroanterior view or posteroanterior and lateral view chest radiography was performed in all patients at the time of TB diagnosis. The presence of cavitation was determined by a radiologist by formally interpreting the chest radiograph. Meanwhile, two of the authors (YS Sim and H Choi) examined the chest radiographs of the patients with cavitary $\mathrm{TB}$ for bilateral involvement, cavity number, and cavity size (the largest size was measured in patients with $\geq 2$ cavities); a consensus was obtained.

\section{Treatment regimen and outcomes}

The standard treatment for drug-susceptible TB is comprised of the intensive phase with daily rifampin, isoniazid, ethambutol, and pyrazinamide for two months, followed by the continuation phase with daily rifampin and isoniazid with or without ethambutol for four months (7). The dosages for each of the medications were determined based on patients' body weight. Patients with body weight $\geq 50 \mathrm{~kg}$ received $600 \mathrm{mg}$ of rifampin, $300 \mathrm{mg}$ of isoniazid, $800 \mathrm{mg}$ of ethambutol, and 1,500 $\mathrm{mg}$ of pyrazinamide $(2,000 \mathrm{mg}$ of pyrazinamide in those with bodyweight $>70 \mathrm{~kg}$ ); those with bodyweight $<50 \mathrm{~kg}$ received $450 \mathrm{mg}$ of rifampin, $300 \mathrm{mg}$ of isoniazid, $800 \mathrm{mg}$ of ethambutol, and 1,000 mg of pyrazinamide according to the Korean guideline for the treatment of TB (12). For patients with high risk for recurrence, including cavitation on chest radiography and positive culture at month 2 , the continuation phase was extended to seven months, corresponding to nine months of total treatment duration. Moreover, the additional factors that should be considered when deciding to prolong treatment in patients with either cavitation or a positive culture at 2 months (but not both) included being $>10 \%$ below ideal body weight; being an active smoker; having diabetes, HIV infection, or any other immunosuppressing condition; or having extensive disease on chest radiograph. In addition, the continuation phase could be extended based on the sputum AFB culture results at the discretion of the attending physicians.

Treatment outcomes were categorized as cured, treatment completion, treatment success, treatment failure, lost to follow-up, not evaluated, and recurrence $(12,13)$. A patient was considered cured if the sputum culture was negative in the last month of treatment and at least two consecutive sputum cultures were negative before the end of treatment. Treatment completion was defined as completion of treatment (at least 180 doses) without the evidence of failure and non-adherence to treatment but not meeting the criteria of being cured. To ensure adherence to treatment in South Korea, through a public-private mix collaboration, the TBspecialist nurses were dispatched to health care facilities. They are responsible for providing patient education and monitoring any adverse effects during treatment. When the patients are lost to regular follow-up, the TB-specialist nurses encourage them to visit the hospital through telephone consultation or home visits $(14,15)$. Treatment success was defined as both cured and completing treatment. Treatment failure was defined as having positive sputum culture results after 4 months of treatment (13). Lost to follow-up was defined as a lack of treatment initiation or treatment interruption for at least two consecutive months. Transferred patients with unknown treatment results were classified as not evaluated. Recurrence was defined as the diagnosis of pulmonary TB in a patient after initial treatment success $(12,13)$. We defined a favorable outcome as treatment success with no evidence of recurrence during the followup period, and an unfavorable outcome as treatment failure or recurrence after initial treatment success. The patients should visit the hospital every 3 months for at least 1 year post-treatment completion to check for recurrence. The primary endpoint of our study was the rate of a favorable outcome.

\section{Statistical analysis}

Data are presented as the median and interquartile range (IQR) for continuous variables and as frequency (percentage) for categorical variables. Data were compared by the MannWhitney $U$ test for continuous variables and by Pearson's chi-square test or Fisher's exact test for categorical variables. To evaluate the effect of a positive culture on the treatment outcomes at month 2, multivariable logistic regression analysis was performed after adjusting for age, sex, body mass index, cavity size, bilateral lung infiltrates, and treatment duration. All tests were two-sided, and $\mathrm{P}$ values of $<0.05$ were considered statistically significant differences. All statistical analyses were performed using IBM SPSS Statistics for Windows (version 23; IBM Corp., Armonk, NY, USA) and 
Table 1 Baseline characteristics of the study population

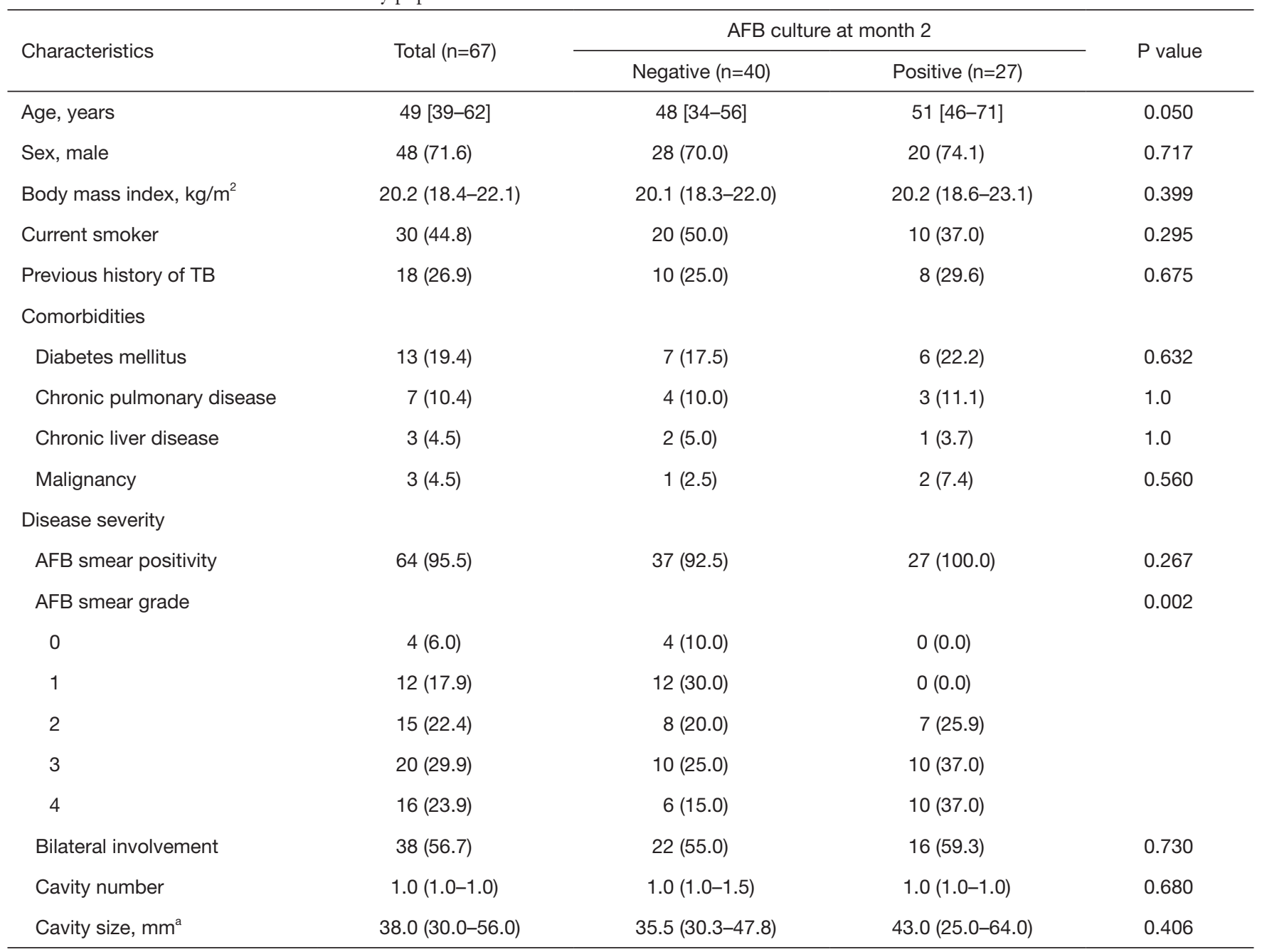

The data are presented as either median and interquartile range or number and percentage, as appropriate. ${ }^{a}$, the largest size was measured in patients with $\geq 2$ cavities. TB, tuberculosis; AFB, acid-fast bacilli.

STATA (version 14; Stata Corp., College Station, TX).

\section{Results}

\section{Patient characteristics}

The clinical characteristics of study participants are summarized in Table 1. None of the patients had HIV infection in this study. Among the 67 patients, 40 (59.7\%) were culture-negative and 27 (40.3\%) were culture-positive after two months of treatment. Patients with positive culture at month 2 showed significantly higher baseline AFB smear grades than those with a negative culture at month $2(\mathrm{P}=0.002)$. Although patients with negative culture at month 2 were younger than those with positive culture at month 2 (48 vs. 51 years), this difference was not significant $(\mathrm{P}=0.050)$. There were no significant differences between the two groups in terms of smoking history, previous history of $\mathrm{TB}$, comorbidities, bilateral lung field involvement on chest radiograph, cavity number, or cavity size on chest radiograph.

\section{Treatment regimen and duration}

Treatment regimen and duration for patients with drugsusceptible cavitary TB are summarized in Table 2. A standard anti-TB medication regimen was initiated in all patients, which consisted of rifampin, isoniazid, ethambutol, and pyrazinamide. Second line anti-TB medications were used in some patients 
Table 2 Treatment regimen and treatment duration

\begin{tabular}{|c|c|c|c|c|}
\hline & Total $(n=67)$ & \multicolumn{2}{|c|}{ AFB culture at month 2} & $P$ value \\
\hline \multicolumn{5}{|l|}{ Regimen of anti-TB drugs } \\
\hline Rifampin & $67(100.0)$ & $40(100.0)$ & $27(100.0)$ & - \\
\hline Isoniazid & $67(100.0)$ & $40(100.0)$ & $27(100.0)$ & - \\
\hline Pyrazinamide & $67(100.0)$ & $40(100.0)$ & $27(100.0)$ & - \\
\hline Fluoroquinolone $^{a}$ & $5(7.5)$ & $4(10.0)$ & $1(3.7)$ & 0.641 \\
\hline Injectable drugs ${ }^{b}$ & $2(3.0)$ & $2(5.0)$ & $0(0.0)$ & 0.512 \\
\hline Cycloserine & $1(1.5)$ & $1(2.5)$ & $0(0.0)$ & 1.0 \\
\hline Isoniazid & $275(256-301)$ & $273(207-284)$ & 279 (264-362) & 0.114 \\
\hline Ethambutol & $271(176-280)$ & $261(177-280)$ & $273(138-280)$ & 0.458 \\
\hline Pyrazinamide & $70(60-87)$ & $70(61-91)$ & 70 (59-83) & 0.450 \\
\hline Fluoroquinolone & $56(14-280)$ & $152(25-296)$ & $14(\mathrm{NA})$ & 0.277 \\
\hline Injectable drugs & 143 (NA) & 143 (NA) & - & - \\
\hline Cycloserine & $14(\mathrm{NA})$ & 14 (NA) & - & - \\
\hline Treatment duration, days & 275 (259-301) & $273(207-284)$ & $279(272-362)$ & 0.066 \\
\hline
\end{tabular}

The data are presented as either median and interquartile range or number and percentage, as appropriate. ${ }^{a}$, three patients received levofloxacin, and two patients received moxifloxacin; ${ }^{b}$, one patient received intramuscular streptomycin, and the other patient received intramuscular streptomycin followed by kanamycin. AFB, acid-fast bacilli; TB, tuberculosis; NA, not applicable.

who had experienced side effects of standard medications, which included a fluoroquinolone in five $(7.5 \%)$ patients (levofloxacin in three and moxifloxacin in two), injectable drugs in two $(3.0 \%)$ (streptomycin in one and streptomycin followed by kanamycin in the other), and cycloserine in one (1.5\%). There were no significant differences in TB treatment regimen between the patients regardless of culture conversion at month 2 after treatment initiation.

Patients were treated with rifampin, isoniazid, and ethambutol for approximately nine months, and pyrazinamide for approximately two months. Although patients with positive culture at month 2 were more likely to be treated for a longer duration, and receive rifampin for a longer duration than in those with negative culture at month 2 , these differences were not significant $(\mathrm{P}=0.066$, both for overall treatment duration and duration of rifampin use).

\section{Treatment outcomes}

As shown in Table 3, the rate of 2-month AFB smear positivity was significantly higher in patients with a positive culture at month 2 than in those with a negative culture at month $2(74.1 \%$ vs. $30.0 \% ; \mathrm{P}=0.001)$. The proportion of patients with treatment success was significantly higher in patients with negative culture at month 2 than in those with a positive culture at month 2 [100\% (40/40) vs. $74.1 \%(20 / 27) ; \mathrm{P}<0.001]$. The reasons for not achieving treatment success in patients with a positive culture at month 2 included treatment failure (18.5\%, 5/27), follow-up loss, or not evaluated (7.4\%, 2/27). In five patients categorized with treatment failure, three had negative cultures in 5 months, one had negative culture in 7 months, and the other had negative culture 9 
Table 3 Treatment outcomes

\begin{tabular}{|c|c|c|c|c|}
\hline Outcomes & Total $(n=67)$ & \multicolumn{2}{|c|}{ AFB conversion at month 2} & $P$ value \\
\hline AFB smear positivity at month 2 & $32(47.8)$ & $12(30.0)$ & $20(74.1)$ & 0.001 \\
\hline Treatment outcomes & & & & 0.001 \\
\hline Cured & $57(85.1)$ & $39(97.5)$ & $18(66.7)$ & \\
\hline Treatment failure $^{a}$ & $5(7.5)$ & $0(0.0)$ & $5(18.5)^{a}$ & \\
\hline Follow-up loss & $1(1.5)$ & $0(0.0)$ & $1(3.7)$ & \\
\hline Not-evaluated & $1(1.5)$ & $0(0.0)$ & $1(3.7)$ & \\
\hline Follow-up duration, months & $15.8(6.3-39.3)$ & $22.3(10.1-41.2)$ & $9.7(3.0-19.3)$ & 0.004 \\
\hline Unfavorable treatment outcomes $^{c}$ & $9(13.4)$ & $1(2.5)$ & $8(29.6)^{a}$ & 0.002 \\
\hline \multicolumn{5}{|l|}{ Development of acquired drug resistance } \\
\hline Rifampin & $1(1.5)$ & $0(0.0)$ & $1(3.7)$ & 0.403 \\
\hline Isoniazid & $1(1.5)$ & $0(0.0)$ & $1(3.7)$ & 0.403 \\
\hline Ethambutol & $0(0.0)$ & $0(0.0)$ & $0(0.0)$ & - \\
\hline Pyrazinamide & $0(0.0)$ & $0(0.0)$ & $0(0.0)$ & - \\
\hline
\end{tabular}

The data are presented as either median and interquartile range or number and percentage, as appropriate. ${ }^{\mathrm{a}}$, one patient who had experienced treatment failure also had a relapse after treatment completion. This case was counted as a single event of an unfavorable outcome; ${ }^{b}$, favorable treatment outcomes were defined as treatment success with no evidence of recurrence during follow-up period; ${ }^{c}$, unfavorable treatment outcomes were defined as treatment failure or recurrence after initial treatment success. AFB, acid-fast bacilli.

months after treatment initiation.

The proportion of patients with favorable treatment outcomes was significantly higher in patients with a negative culture at month 2 than in those with a positive culture [97.5\% (39/40) vs. 70.4\% (19/27); $\mathrm{P}=0.002]$. The recurrence rate after treatment success was $2.5 \%(1 / 40)$ in patients with a negative culture at month 2 and $8 \%(2 / 25)$ in those with a positive culture at month 2, respectively $(\mathrm{P}=0.554)$. The median follow-up duration post-treatment completion was 15.8 months (IQR, 6.3-39.3 months). Each follow-up duration was 22.3 months (IQR, 10.1-41.2 months) in patients with a negative culture at month 2, and 9.3 months (IQR, 3.0-19.3 months) in those with a positive culture at month 2 , respectively $(\mathrm{P}=0.004)$. Among patients with negative culture at month 2, one patient had recurrence after treatment success. The patient was a 49 -year-old man with a low BMI $\left(17.4 \mathrm{~kg} / \mathrm{m}^{2}\right)$, current smoking history, and bilateral disease on the initial chest radiograph.

\section{The effect of positive culture at month 2 on treatment outcomes}

As shown in Table 4, regardless of the definition of unfavorable treatment outcome, patients with positive culture at month 2 were more likely to have unfavorable treatment outcomes than those with negative culture at month 2 (unadjusted OR, 16.42, 95\% CI, 1.91-140.07; adjusted OR, 17.04, 95\% CI, 1.68-172.92 for unfavorable outcomes defined as treatment failure, follow-up loss, not evaluated, or recurrence after initial treatment success, unadjusted OR, 12.32, 95\% CI, 1.38-109.71; adjusted OR,18.69, 95\% CI, 1.05-333.05 for unfavorable outcomes defined as treatment failure or recurrence after initial treatment success).

\section{Disease course of patients with initial treatment failure}

Clinical characteristics and treatment regimens of five patients with initial treatment failure are provided in 
Table 4 Crude and adjusted odds ratio for the unfavorable treatment outcomes in patients with positive culture at month 2 over those with negative culture at month 2

\begin{tabular}{|c|c|c|c|c|c|c|}
\hline & \multicolumn{3}{|c|}{ AFB culture at month $2^{a}$} & \multicolumn{3}{|c|}{ AFB culture at month $2^{\mathrm{b}}$} \\
\hline Crude model & References & $16.42(1.91-140.07)$ & 0.011 & References & $12.32(1.38-109.71)$ & 0.024 \\
\hline Adjusted model ${ }^{c}$ & References & $17.04(1.68-172.92)$ & 0.016 & References & $18.69(1.05-333.05)$ & 0.046 \\
\hline
\end{tabular}

The patient who had both treatment failure and recurrence after treatment completion was counted as one case. ${ }^{a}$, unfavorable outcomes were defined as treatment failure, follow-up loss/not evaluated, or recurrence after initial treatment success; ${ }^{b}$, unfavorable outcomes were defined as treatment failure or recurrence after initial treatment success, except for those with follow-up loss $/$ not evaluated $(n=2) ;{ }^{\circ}$, age, sex, body mass index, cavity size, bilateral lung infiltrates, and treatment duration were factors in the adjusted model. AFB, acid-fast bacilli.

Supplementary Table S1. DST performed using $M$. tuberculosis grown on month 4 sputum cultures did not reveal any new drug resistance. After detecting treatment failure, all five patients received a treatment regimen composed of isoniazid and rifampin with or without ethambutol, which was the standard continuation phase regimen. Three patients achieved culture conversion after extending the duration of therapy, one patient developed MDR-TB at 5.5 months after treatment initiation, and one patient was lost to follow-up 5.8 months after culture conversion, which was achieved 8.7 months from treatment initiation. Of the three patients who had achieved treatment success after prolonged treatment, recurrence was observed in one patient 37.7 months after treatment completion.

\section{Discussion}

The present study evaluated treatment outcomes of an extended treatment regimen of 67 patients with drugsusceptible cavitary pulmonary TB. At month 2, two-thirds of patients had negative sputum culture, and one-third had positive sputum culture. The rate of favorable treatment outcomes were significantly higher in patients with negative culture at month 2 compared to those with a positive culture. The extended treatment regimen showed favorable treatment outcomes in patients with a negative culture at month 2; however, it might not be enough to overcome unfavorable outcomes in those with a positive culture at month 2.

Several studies using qualitative smears and cultures have shown that patients with cavitary TB have higher mycobacterial loads in their sputum (16-20), which may result in a high recurrence rate, ranging up to $20 \%$ in cavitary TB patients, especially in those with a positive culture at month 2 (7-9). These study results suggest that the current six-month standard therapy is not an adequate treatment duration for cavitary pulmonary TB despite drug-susceptibility. Thus, the current treatment guidelines recommend extending the continuation phase therapy for three months in cavitary TB patients with a positive culture at month 2, and considering an extended continuation phase therapy in TB patients with only cavitation (7). However, there have been insufficient data to support this recommendation.

From this perspective, our study provided informative data about the treatment outcomes of an extended treatment regimen in these patients. Supporting the current expert recommendation, the recurrence rate after extended treatment was about $4.6 \%$ (2.5\% in patients with a negative culture at month 2 and $8.0 \%$ in patients with a positive culture at month 2) in this study, which is lower than the previous results (ranging up to $20 \%$ ) in patients who received a six-month treatment (8). The factors related to recurrence including age, underlying comorbidities, and disease severity in this study were comparable with those of the previous study conducted by Jo and colleagues (8). Moreover, a recent meta-analysis reported that the subgroup of patients with both cavitation and positive 2 -month smear positivity had shown a relapse risk of at least $10 \%$ (21). Thus, the extended treatment duration may have a significant role in decreasing the recurrence rate. In addition, the Korean Government public-private mix collaboration policy (explained in the Methods section in detail) $(14,15)$, which has been settled since 2011, may have an additional role in decreasing the recurrence rate by enhancing medication adherence.

Unfortunately, the treatment success rate was significantly different depending on the culture results at month 2 . While patients with negative culture at month 2 showed a high treatment success rate (100\%), comparable with those 
of drug-susceptible TB in previous reports $(1,22)$, patients with positive culture at month 2 showed only a $74 \%$ treatment success rate. Thus, due to a high treatment failure rate, despite the low recurrence rate, it could be postulated that cavitary pulmonary $\mathrm{TB}$ with positive culture at month 2 may not be successfully treated by only extending the duration of continuation therapy. Previous studies have also shown that positive smear and/or culture at month 2 (23-25) is associated with treatment failure. Although a more severe disease condition, i.e., the presence of cavitation and positive culture at month 2 , is suggested to be associated with a higher risk of treatment failure, few data have been available; a previous study showed that treatment failure and recurrence rates are higher in patients with both cavitation and positive culture at month 2 ; however, the study did not separately evaluate treatment failure from recurrence (2). To the best of our knowledge, this is the first study showing that both the presence of cavitation and positive culture at month 2 are significantly associated with treatment failure.

In this study, five patients among those with positive culture at month 2 experienced treatment failure, defined as persistently positive culture results after four months of treatment $(7,12)$. Although maintaining the continuation phase therapy led to culture conversion in three patients, one patient was lost to follow-up, and one patient acquired MDR-TB. Some researchers have raised concerns that lengthy TB treatment might increase the development of drug resistance $(26,27)$. Supporting this perspective, one fifth developed MDR-TB during extended treatment for cavitary TB in this study. Thus, although it could be postulated that extending the continuation phase therapy might be helpful to reduce the recurrence rate, we should be careful not to facilitate the development of MDR-TB. We believe that reinforced 2 -month intensive phase regimen may shorten the treatment duration as well as prevent the development of drug resistance in patients with unfavorable outcomes. Future research highlighting new regimens is needed to overcome the drug resistance issue.

Our results, along with the findings of previous studies, strongly suggest that an augmented treatment regimen may be needed for the treatment of cavitary pulmonary TB in patients with positive culture at month 2 . Considering the poor penetration of first line oral anti-TB drugs in cavitary lesions (28-30), augmentation of the treatment regimen with second line drugs such as moxifloxacin may be helpful (29). A previous study using an animal model revealed that moxifloxacin showed a high tissue-plasma ratio of distribution in cavitary lesions (nine in cavity caseum and 16 in the cavity wall), while tissue-plasma ratios of isoniazid, rifampin, and pyrazinamide were relatively unsatisfactory (29). In addition, a recently published article by Strydom and colleagues investigated TB drugs' distribution in the patient's lung lesions and suggested a mechanistic model for regimen and dose optimization (31). We may incorporate such a promising tool into practice, which will lead to more tailored regimen to overcome subtherapeutic drug concentrations in cavities.

The extended continuation phase was an acceptable strategy for the treatment of cavitary pulmonary TB patients with negative culture at month 2 . Since treatment outcomes were much better in cavitary TB patients with negative culture at month 2 than those with positive culture at month 2, we could postulate that a six-month standard regimen may be enough for some of the patients with negative culture at month 2 . However, as all patients with cavitary TB received extended treatment in this study, we could not evaluate the treatment outcomes of the standard six-month therapy. Thus, further studies comparing the treatment outcomes of standard six-month therapy versus extended continuation phase therapy in cavitary pulmonary TB are needed.

This study has several limitations. First, it was a retrospective study with a relatively small sample size from a single referral hospital. For example, cavity size was larger in patients with a positive culture at month 2 than in those with a negative culture at month 2 , which might have resulted in culture positivity at month 2 and hence treatment failure. However, the difference in cavity size was not statistically significant, probably due to the small sample size. Second, because genotyping was not available, this study could not discriminate relapse due to reactivation from recurrence due to exogenous reinfection (32). Third, although there was no significant difference in the recurrence rate between patients with positive and negative culture at month 2 , the statistical insignificance may be due to the relatively small study population. However, this limitation does not change our study findings regarding the potential role of an extended continuation regimen. Studies with a larger number of patients are needed to confirm these findings.

In conclusion, the culture result at month 2 is the most critical factor affecting treatment outcomes in patients with drug-susceptible cavitary TB. Patients with negative culture at month 2 showed favorable treatment outcomes comparable with those of drug-susceptible $\mathrm{TB}$ without cavitation. In contrast, patients with positive culture at 
month 2 showed unfavorable treatment outcomes with a higher treatment failure rate, which was not overcome by merely extending the continuation phase. This study suggests that an augmented treatment regimen might be needed in cavitary pulmonary TB patients with positive culture at month 2 .

\section{Acknowledgments}

Funding: This research was supported by Basic Science Research Program through the National Research Foundation of Korea (NRF) funded by the Ministry of Science, Information and Communications Technologies (NRF-2019R1G1A1008692).

\section{Footnote}

Conflicts of Interest: The authors have no conflicts of interest to declare.

Ethical Statement: The authors are accountable for all aspects of the work in ensuring that questions related to the accuracy or integrity of any part of the work are appropriately investigated and resolved. The study protocol was approved by the Institutional Review Board (IRB) of Hallym University Kangnam Sacred Heart Hospital (IRB application no. 2018-07-007).

Open Access Statement: This is an Open Access article distributed in accordance with the Creative Commons Attribution-NonCommercial-NoDerivs 4.0 International License (CC BY-NC-ND 4.0), which permits the noncommercial replication and distribution of the article with the strict proviso that no changes or edits are made and the original work is properly cited (including links to both the formal publication through the relevant DOI and the license). See: https://creativecommons.org/licenses/by-nc-nd/4.0/.

\section{References}

1. Zumla A, Raviglione M, Hafner R, et al. Tuberculosis. N Engl J Med 2013;368:745-55.

2. Baker MA, Harries AD, Jeon CY, et al. The impact of diabetes on tuberculosis treatment outcomes: a systematic review. BMC Med 2011;9:81.

3. Chang KC, Leung CC, Yew WW, et al. A nested casecontrol study on treatment-related risk factors for early relapse of tuberculosis. Am J Respir Crit Care Med
2004;170:1124-30.

4. Horne DJ, Royce SE, Gooze L, et al. Sputum monitoring during tuberculosis treatment for predicting outcome: systematic review and meta-analysis. Lancet Infect Dis 2010;10:387-94.

5. Khan A, Sterling TR, Reves R, et al. Lack of weight gain and relapse risk in a large tuberculosis treatment trial. Am J Respir Crit Care Med 2006;174:344-8.

6. Wang JY, Lee MC, Shu CC, et al. Optimal duration of anti-TB treatment in patients with diabetes: nine or six months? Chest 2015;147:520-8.

7. Nahid P, Dorman SE, Alipanah N, et al. Executive Summary: Official American Thoracic Society/Centers for Disease Control and Prevention/Infectious Diseases Society of America Clinical Practice Guidelines: Treatment of Drug-Susceptible Tuberculosis. Clin Infect Dis 2016;63:853-67.

8. Jo KW, Yoo JW, Hong Y, et al. Risk factors for 1-year relapse of pulmonary tuberculosis treated with a 6-month daily regimen. Respir Med 2014;108:654-9.

9. Benator D, Bhattacharya M, Bozeman L, et al. Rifapentine and isoniazid once a week versus rifampicin and isoniazid twice a week for treatment of drug-susceptible pulmonary tuberculosis in HIV-negative patients: a randomised clinical trial. Lancet 2002;360:528-34.

10. Diagnostic Standards and Classification of Tuberculosis in Adults and Children. This official statement of the American Thoracic Society and the Centers for Disease Control and Prevention was adopted by the ATS Board of Directors, July 1999. This statement was endorsed by the Council of the Infectious Disease Society of America, September 1999. Am J Respir Crit Care Med 2000;161:1376-95.

11. Bai GH, Park YK, Choi YW, et al. Trend of antituberculosis drug resistance in Korea, 1994-2004. Int J Tuberc Lung Dis 2007;11:571-6.

12. Joint Committee for the Revision of Korean Guidelines for Tuberculosis Korea Centers for Disease Control and Prevention. Korean Guidelines for Tuberculosis. 2017.

13. Centers for Disease Control and Prevention. Treatment of Tuberculosis, American Thoracic Society, CDC, and Infectious Diseases Society of America. 2003;52.

14. Cho KS. Tuberculosis control in the Republic of Korea. Epidemiol Health 2018;40:e2018036-0.

15. Noh CS, Kim HI, Choi H, et al. Completion rate of latent tuberculosis infection treatment in patients aged 65 years and older. Respir Med 2019;157:52-8.

16. Palaci M, Dietze R, Hadad DJ, et al. Cavitary disease and 
quantitative sputum bacillary load in cases of pulmonary tuberculosis. J Clin Microbiol 2007;45:4064-6.

17. Gomes M, Saad Junior R, Stirbulov R. Pulmonary tuberculosis: relationship between sputum bacilloscopy and radiological lesions. Rev Inst Med Trop Sao Paulo 2003;45:275-81.

18. Matsuoka S, Uchiyama K, Shima H, et al. Relationship between CT findings of pulmonary tuberculosis and the number of acid-fast bacilli on sputum smears. Clin Imaging 2004;28:119-23.

19. Rathman G, Sillah J, Hill PC, et al. Clinical and radiological presentation of 340 adults with smear-positive tuberculosis in The Gambia. Int J Tuberc Lung Dis 2003;7:942-7.

20. Murthy SE, Chatterjee F, Crook A, et al. Pretreatment chest $\mathrm{x}$-ray severity and its relation to bacterial burden in smear positive pulmonary tuberculosis. BMC Medicine 2018;16:73.

21. Romanowski K, Balshaw RF, Benedetti A, et al. Predicting tuberculosis relapse in patients treated with the standard 6-month regimen: an individual patient data meta-analysis. Thorax 2019;74:291-7.

22. Chang KC, Leung CC, Yew WW, et al. Dosing schedules of 6-month regimens and relapse for pulmonary tuberculosis. Am J Respir Crit Care Med 2006;174:1153-8.

23. Dembele SM, Ouedraogo HZ, Combary A, et al. Conversion rate at two-month follow-up of smear-positive tuberculosis patients in Burkina Faso. Int J Tuberc Lung Dis 2007;11:1339-44.

24. Singla R, Bharty SK, Gupta UA, et al. Sputum smear positivity at two months in previously untreated pulmonary tuberculosis patients. Int J Mycobacteriol 2013;2:199-205.

25. Pefura-Yone EW, Kengne AP, Kuaban C. Non-conversion of sputum culture among patients with smear positive pulmonary tuberculosis in Cameroon: a prospective cohort study. BMC Infectious Diseases 2014;14:138.

26. Senousy BE, Belal SI, Draganov PV. Hepatotoxic effects of therapies for tuberculosis. Nat Rev Gastroenterol Hepatol 2010;7:543-56.

27. de Steenwinkel JE, ten Kate MT, de Knegt GJ, et al. Consequences of noncompliance for therapy efficacy and emergence of resistance in murine tuberculosis caused by the Beijing genotype of Mycobacterium tuberculosis. Antimicrob Agents Chemother 2012;56:4937-44.

28. Barclay WR, Ebert RH, Le Roy GV, et al. Distribution and excretion of radioactive isoniazid in tuberculous patients. J Am Med Assoc 1953;151:1384-8.

29. Kjellsson MC, Via LE, Goh A, et al. Pharmacokinetic evaluation of the penetration of antituberculosis agents in rabbit pulmonary lesions. Antimicrob Agents Chemother 2012;56:446-57.

30. Prideaux B, Via LE, Zimmerman MD, et al. The association between sterilizing activity and drug distribution into tuberculosis lesions. Nat Med 2015;21:1223-7.

31. Strydom N, Gupta SV, Fox WS, et al. Tuberculosis drugs' distribution and emergence of resistance in patient's lung lesions: A mechanistic model and tool for regimen and dose optimization. PLoS Med 2019;16:e1002773.

32. Wang JY, Lee LN, Lai HC, et al. Prediction of the tuberculosis reinfection proportion from the local incidence. J Infect Dis 2007;196:281-8.
Cite this article as: Lee H, Sohn JW, Sim YS, Shin TR, Kim DG, Choi H. Outcomes of extended duration therapy for drug-susceptible cavitary pulmonary tuberculosis. Ann Transl Med 2020;8(6):346. doi: 10.21037/atm.2020.02.104 


\section{Supplementary}

Table S1 Clinical characteristics and treatment regimens in five patients with initial treatment failure

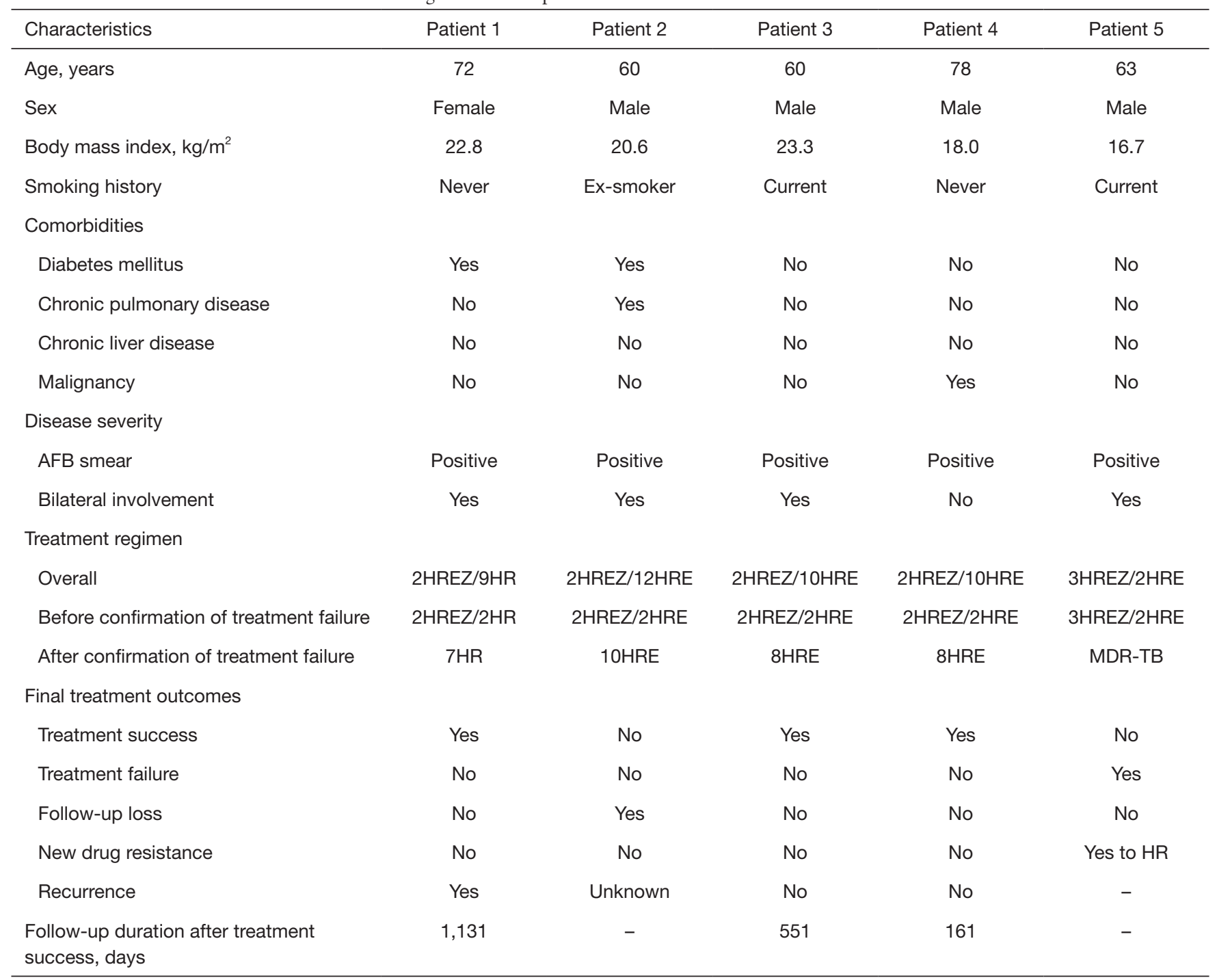

AFB, acid-fast bacilli; H, isoniazid; R, rifampin; E, ethambutol; Z, pyrazinamide; MDR, multidrug-resistant; TB, tuberculosis. 